\title{
Robotic Search for Optimal Cell Culture in Regenerative Medicine
}

\author{
Genki N. Kanda ${ }^{1,2,3, *, \boxminus}$, Taku Tsuzuki ${ }^{4, *}$, Motoki Terada ${ }^{1}$, Noriko Sakai ${ }^{1,6}$, Naohiro Motozawa ${ }^{1}$, Tomohiro \\ Masuda ${ }^{1}$, Mitsuhiro Nishida ${ }^{1}$, Chihaya T. Watanabe ${ }^{4}$, Tatsuki Higashi ${ }^{4}$, Shuhei A. Horiguchi ${ }^{4}$, Taku Kudo ${ }^{3}$, \\ Motohisa Kamei ${ }^{3}$, Genshiro A. Sunagawa ${ }^{1}$, Kenji Matsukuma ${ }^{3}$, Takeshi Sakurada ${ }^{4}$, Yosuke Ozawa ${ }^{4, \boxminus}$, Masayo \\ Takahashi $^{1,6}$, Koichi Takahashi ${ }^{2, \boxminus}$, Tohru Natsume ${ }^{3,5, \boxminus}$ \\ ${ }^{1}$ Laboratory for Retinal Regeneration, RIKEN Center for Biosystems Dynamics Research. \\ 2 Laboratory for Biologically Inspired Computing, RIKEN Center for Biosystems Dynamics Research. \\ ${ }^{3}$ Robotic Biology Institute Inc. \\ ${ }^{4}$ Epistra Inc. \\ ${ }^{5}$ Department of Life Science and Biotechnology, Cellular and Molecular Biotechnology Research Institute, National \\ Institute of Advanced Industrial Science and Technology. \\ ${ }^{6}$ Vision Care Inc. \\ * Co-First authors (equal contribution), ${ }^{\circledR}$ Contact authors
}

Lead contact: Tohru Natsume, Ph.D. (t-natsume@aist.go.jp)

\begin{abstract}
Induced differentiation is one of the most experience- and skill-dependent processes in regenerative medicine, and establishing optimal conditions often takes years (an inordinate amount of time). Here, we developed a robotic-Al system that autonomously induces the differentiation of iPS cell-derived RPE (iPSC-RPE) cells. The system performed 216 forty-day cell culture experiments, with a total experimentation time of 8,640 days. The search for optimal differentiation conditions was accelerated using a novel batch Bayesian optimization technique with local penalization, compressing the search time to 185 days, with a cumulative robot operating time of $995 \mathrm{~h}$. From 200 million possible parameter combinations, the system optimized the iPSC-RPE production conditions to yield improved pigmented scores that were up to $88 \%$ higher than the scores obtained with the pre-optimized conditions. Transferring tacit knowledge and skills often constitutes a serious obstacle when transposing basic cell experimental research from the laboratory to the medical forefront or for scaling to mass production. Our work demonstrates that autonomous robotic-Al systems can be effectively utilized for the systematic exploration of experimental conditions independently from the tacit knowledge of skilled professionals. This option guarantees immense use in future research.
\end{abstract}

\section{INTRODUCTION}

Automating scientific discovery is one of the grandest challenges of the 21st century (1). A promising approach consists in creating a closed loop of computation and experimentation by combining $\mathrm{Al}$ and robotics (2). A relatively simple form of autonomous knowledge discovery consists in searching for optimal experimental procedures and parameter sets through repeated experimentation and result validation according to a predefined validation method. As an example, in material science, the parameters involved in the growth of carbon nanotubes were explored using an autonomous closedloop learning system (3). Moreover, in experimental physics, Bayesian optimization has been used to identify the optimal evaporation ramp conditions for BoseEinstein condensate production (4). In 2019, the promoter combination search in molecular biology was automated using an optimization algorithm-driven robotic system (5).

Here, we report the development of a robotic search system that autonomously determines optimal conditions in cell culture. Cell culture is probably one of the most delicate procedures, as it deals with living entities that have very complicated internal states. High-end regenerative medical cells need to be artificially differentiated from ES/iPS cells to achieve functional recovery of tissues and individuals. The complex internal state of cells prevents the determination of uniform cell culture conditions in these subjects, and has traditionally required humans to manually search for the suitable culture conditions of each strain and/or lot (6). We created a digital representation of the high-end regenerative medical cell culture protocol used for the induction of 
retinal pigment epithelial (RPE) cell differentiation from iPS cells (iPSC-RPE cell) (7) that can be executed by Maholo LabDroids. LabDroid is a versatile humanoid robot that can perform a broad range of cell culture (8). The robot was combined with an Al system that evaluates the experimental results and autonomously searches for optimal conditions with batch Bayesian optimization (BBO; Figure 1). In this paper we first describe the robotization of the iPSC-RPE differentiation protocol with a LabDroid. Second, we explain the parameterization of the iPSC-RPE protocol and the novel optimization method. Finally, we prove that iPSC-RPE cells generated by LabDroid satisfy the cell biological criteria for regenerative medicine research applications.

\section{RESULTS}

\section{Robotization of the iPSC-RPE differentiation protocol}

An overview of the iPSC-RPE differentiation protocol used for the optimization target is shown in Figure 2A. It consists of five steps: seeding, preconditioning, passage, induction of RPE differentiation (induction), and RPE maintenance culture. The day on which the passage was performed was defined as differentiation day (DDay) 0 , and the cultured cells were sampled and validated on DDays 33 and 34 . To conduct this protocol with the LabDroid, the necessary peripherals are installed on and around the LabDroid's workbench (Figure 2B). We designed the system to work simultaneously with eight 6well plates per batch (48 cell-containing wells in total). LabDroid was programmed for three types of operations: seeding, medium exchange, and passage (Figure $\mathbf{2 C}-\mathbf{F}$ ). The steps for preconditioning and induction of RPE differentiation, which correspond to the preparation of reagents, were named medium exchange type I (Figure 2A, D), and the step for RPE maintenance culture, which does not involve reagent preparation, was named medium exchange type II (Figure 2A, E).

First, we used LabDroid to perform iPSC-RPE induced differentiation under the same conditions as the typical manual operations (baseline experiments). Because of the differences in structure and experimental environment between LabDroid and humans, some operations and/or movements such as the use of a centrifuge, the presence or absence of cell counting at the time of passage, and the speed of movement, differ from those of humans. For example, achieving the same time interval for trypsin treatment in all wells of a single plate during cell detachment using LabDroid becomes rather difficult. Therefore, the passage operation was performed in six separate time intervals. The cells differentiating into RPE produce melanin, which causes them to turn brown. Therefore, the area ratio of the total number of pigmented cells on DDay 34 was used to estimate the differentiation induction efficiency and obtain evaluation scores. These validation scores were used to simplify the validation process and did not reflect the entire quality of the RPE.

Baseline experiments were conducted and validated using a total of 6 trypsin conditions and 8 plates (Figure 3). The highest-scoring trypsin treatment was $20 \mathrm{~min}$ at $37^{\circ} \mathrm{C}$, followed by $14 \mathrm{~min}$ incubation at room temperature, with an 8-plate score of $0.44 \pm 0.03$ (mean \pm SEM, $n=8$ ). The lowest-scoring trypsin treatment of the six trypsin conditions was incubation for $20 \mathrm{~min}$ at $37^{\circ} \mathrm{C}$ followed by $23 \mathrm{~min}$ at room temperature, with an 8-plate score of 0.33 \pm 0.02 (mean \pm SEM, $n=8$ ). LabDroid successfully performed the iPSC-RPE protocol as evidenced by the detection of pigmented cells in all 48 wells and the lack of errors in the operating process. However, the efficiency of induction was insufficient. Therefore, we attempted to optimize the protocol parameters to further improve the scores.

\section{Parameterization of protocol}

To improve the pigmented score, we selected seven parameters for optimization: two from the preconditioning step, three from the passage step, and two from the RPE differentiation induction step. Search domains were then set for each parameter (Table 1; Figure 4A, B).

From the preconditioning step on DDays -1 to -6 , we selected two parameters to optimize: the concentration of FGF receptor inhibitor (FGFRi) in the medium ( $P C$, preconditioning concentration), and the duration of addition ( $P P$, preconditioning period). From the passage step performed on DDay 0 , we selected three parameters to optimize: the pipetting strength during cell detachment (DS, detachment pipetting strength), the area of the bottom surface to be pipetted $(D L$, detachment pipetting length), and the trypsin processing time ( $D P$, detachment trypsin period) at a passage. $D P$ is a contextualparameter that can only be used to perform experiments at fixed values due to the experimental system's specifications. In this case, $D P$ is allowed to take different fixed values at three-minute intervals corresponding to the number of wells in the same plate. From the RPE differentiation induction step on DDays 1 to 25, we selected two parameters to optimize: the concentration of $\mathrm{KSR}$ in the medium (KP, KSR period), and the duration of exposure period of the three chemical supplements $(3 P$, three supplements period).

\section{Optimization of protocol}

To improve optimization performance, 48 conditions (8 plates $\times 6$ wells described in Figure $3 \mathbf{A}$ ) in parallel in one batch were executed. In general, solving a highdimensional and expensive black box optimization problem like the present one with a limited number of rounds poses quite a challenge. In our case, some 200 million possible parameter combinations exist in the search space, and the point where the pigmented score is optimal in 3 rounds ( 144 queries) had to be determined because one experiment round takes 40-45 days. In recent studies, batch Bayesian optimization (BBO) has 
evidenced excellent performance concerning such realworld black-box optimization problems $(5,9,10)$. We developed an experimental design module based on batch Bayesian optimization to effectively search for the optimal experimental parameter that maximizes the pigmented scores in the search space defined in Figure 4B.

The Bayesian optimization module generates queries using two components: the Model updater which updates the surrogate model that captures the relationship between parameters and the scores using Bayesian inference; and the Query generator that generates the next experimental parameters $\boldsymbol{X}_{\text {next }}$ using an acquisition function and a policy function (Figure 5A). In the Query generator, the acquisition function estimates the expected progress toward the optimal experimental parameter at a given experimental parameter (Figure 5B). Then, using the acquisition function, the policy function generates the next 48 experimental parameters $\boldsymbol{X}_{\text {next }}$ considering contexts regarding the trypsin processing time $x_{\mathrm{DP}}$ (Figure $\mathbf{5 C}$ ).

To test the performance of the Bayesian optimization module in our case, we executed a preliminary performance validation using a toy testing function constructed on domain knowledge.

\section{Robotic optimization drastically improved pigmented score}

In this study, three successive experiments are conducted to optimize the target protocol. In round 1, 48 conditions were generated on the basis of a uniform distribution and translated to LabDroid operating programs. In accordance with the experiment design, we incorporated the two highest-scoring conditions from the previous experiment (Figure 2C) as control conditions, performed differentiation-inducing cultures with LabDroid, and validated the area of the colored cells. Although one condition was found to be experimentally deficient, the other 47 conditions were validated. The highest score was 0.86 (Figure 6A), yielding five conditions that exceeded the mean value (0.39) for all wells in the baseline experiment (Figure 2 C). In round 2, 46 conditions were generated with Bayesian optimization and the two highest-scoring conditions in round 1 were incorporated as control conditions. The highest score was 0.83 (Figure 6B). In round 3 , a second Bayesian optimization was conducted, yielding the improved highest score of 0.91 . We obtained 26 other conditions that were better than the highest one in round 2 (Figure 6C). A visualization diagram of a two-dimensional partial least squares regression (PLS) clearly revealed that the overall experimental parameters tend to converge in a higher pigmented score direction from rounds 1 to 3 (Figure 6D).

To determine whether the optimized conditions were statistically improved over the pre-optimized conditions, a multi-well validation experiment was conducted using the top five conditions in round 3 and the pre-optimized conditions. The validation values were $0.71 \pm 0.06,0.72$ $\pm 0.03,0.76 \pm 0.02,0.79 \pm 0.02$, and $0.81 \pm 0.02$ in the $1 \mathrm{st}, 2 \mathrm{nd}, 3 \mathrm{rd}, 4 \mathrm{th}$, and 5 th place, respectively (mean \pm $\mathrm{SEM}, \mathrm{n}=3$ each). All scores were statistically significantly higher than the pre-optimization scores of $0.43 \pm 0.02$ (mean \pm SEM, $n=3$ ) (Figure 7A, B).

To confirm whether the obtained iPSC-RPE cells had the functional characteristics of RPE cells, cells from some of the wells in the top five conditions of round 3 were manually purified, stored, re-cultured, and analyzed. The analyzed iPSC-RPE cells expressed BEST1, RPE65, and CRALBP (Figure 7C), which are characteristic marker genes of RPE cells. In addition, the secretion of VEGF and PEDF in the culture medium, a characteristic of RPE cells, was observed (Figure 7D, E). The expression of tight junction-associated factor ZO-1 was examined using immunohistochemistry, and a ZO-1derived fluorescence signal was observed in microphthalmia-associated transcription factor (MITF) positive cells, which plays a central role in function of RPE cells (Figure 7F). These results indicated that the generated iPSC-RPE cells have the characteristics of RPE cells, and fulfil the criteria for use in regenerative medicine research in the type of analysis measured here (7).

In summary, we conducted 216 forty-day cell culture experiments, with a total experimentation time of 8640 days. We accelerated the search by a batch Bayesian optimization technique, compressing the search time to 185 days with a cumulative robot operating time of 995 hours.

\section{DISCUSSION}

Laboratory automation is a recently developing technology that transfers human skills to machines. Although some robotic systems for cell culture have already been developed (11-18), many of these fixedprocess automation apparatuses lack the flexibility and the precision necessary to execute comprehensive parameter searching. Biological cells are physical systems with rather rich internal dynamics (19) and are less tolerant to differences in manufacturing processes than products derived from metal fabrication, chemical synthesis, or other similar areas (20), underscoring the need for closed-loop optimization.

Combining a LabDroid and a batch Bayesian optimization algorithm, our robotic search system autonomously discovered optimal conditions that improved the efficiency of differentiation induction in iPSC-RPE production by up to $88 \%$ (Figure 7A). We chose the iPSC-RPE differentiation induction protocol for three reasons: first, melanin pigmentation is a single, easily measurable cell morphological indicator of quality and quantity of successfully differentiated RPE cells. The pigmentation score is a fairly well established measure of RPE differentiation quality that can be easily verified with the naked eye. Integrating other modality of data such as 
the RNA expression and secretory protein data shown in Figure 7 C-G to the scoring function would greatly improve the accuracy of iPSC-RPE cells quality estimation. Second, the operating accuracy and repeatability of the robotic system used in this study were satisfactory for the efficient completion of the search process. The iPSC-RPE cell differentiation protocol requires 40 days to run, and a single misoperation, error, or inaccuracy can deteriorate search efficiency, if not destroy the entire process. Third, iPSC-RPE cells have already been clinically transplanted to human patients, and a well-established protocol is available (7). Data and expertise accumulated in manual differentiation induction experiments provide useful information for establishing the general structure of the protocol and in defining its parameter search space. However, many other cell types and differentiation targets lack established protocols, some of which probably demand a more sophisticated optimization technique able to deal with categorical values and their combinations. Such technique could optimize the structure of the protocol simultaneously with continuous parameter values while minimizing the execution costs incurred by the large number of possible combinations (21).

\section{MATERIALS AND METHODS}

\section{Guidelines}

All experiments that involved the use of humanderived samples were reviewed and approved by the institutional review board at the Institutional Committee of RIKEN Kobe Branch.

\section{Reagents}

hiPSC maintenance medium: $80 \%$ StemFit Basal Solution A and $20 \%$ StemFit iPS Expansion Solution B (\#AK02N, Ajinomoto Co., Inc., Japan)

RPE differentiation medium (20\% KSR): $0.10 \mathrm{mM}$ MEM Non-Essential Amino Acids Solution (NEAA) (\#11140050, Thermo Fisher Scientific Inc., MA, USA), 1.0 mM Sodium pyruvate (\#S8636, Merck \& Co., Inc., NJ, USA), 19\% KnockOut ${ }^{\mathrm{TM}}$ Serum Replacement (KSR) (\#10828028, Thermo Fisher Scientific Inc., MA, USA), 0.0007\% 2-mercaptoethanol (\#139-06861, FUJIFILM Wako Pure Chemical Corporation, Japan), $78 \mathrm{U} / \mathrm{mL}$ Benzylpenicillin Sodium, and $78 \mu \mathrm{g} / \mathrm{mL}$ Streptomycin sulfate (\#15140122, Thermo Fisher Scientific Inc., MA, USA). All diluted in GMEM (\#11710035, Thermo Fisher Scientific Inc., MA, USA)

RPE differentiation medium (15\% KSR): $0.10 \mathrm{mM}$ MEM Non-Essential Amino Acids Solution (NEAA) (\#11140050, Thermo Fisher Scientific Inc., MA, USA), 0.99 mM sodium pyruvate (\#S8636, Merck \& Co., Inc., NJ, USA), 15\% KnockOut ${ }^{\mathrm{TM}}$ Serum Replacement (KSR) (\#10828028, Thermo Fisher Scientific Inc., MA, USA), 0.0007\% 2-mercaptoethanol (\#139-06861, FUJIFILM Wako Pure Chemical Corporation, Japan), $82 \mathrm{U} / \mathrm{mL}$ benzylpenicillin sodium, and $82 \mu \mathrm{g} / \mathrm{mL}$ streptomycin sulfate (\#15140122, Thermo Fisher Scientific Inc., MA, USA). All diluted in GMEM (\#11710035, Thermo Fisher Scientific Inc., MA, USA)

RPE differentiation medium (10\% KSR): $0.094 \mathrm{mM}$ MEM Non-Essential Amino Acids Solution (NEAA) (\#11140050, Thermo Fisher Scientific Inc., MA, USA), $0.94 \mathrm{mM}$ sodium pyruvate (\#S8636, Merck \& Co., Inc., NJ, USA), 10\% KnockOut TM Serum Replacement (KSR) (\#10828028, Thermo Fisher Scientific Inc., MA, USA), 0.0007\% 2-mercaptoethanol (\#139-06861, FUJIFILM Wako Pure Chemical Corporation, Japan), $85 \mathrm{U} / \mathrm{mL}$ benzylpenicillin sodium, and $85 \mu \mathrm{g} / \mathrm{mL}$ streptomycin sulfate (\#15140122, Thermo Fisher Scientific Inc., MA, USA). All diluted in GMEM (\#11710035, Thermo Fisher Scientific Inc., MA, USA)

RPE maintenance medium: 29\% Nutrient Mixture F12 (\#N6658, Merck \& Co., Inc., NJ, USA), 1.9 mM LGlutamine (\#G7513, Merck \& Co., Inc., NJ, USA), 1.9\% B-27 ${ }^{\text {TM }}$ supplement, serum free $(\# 17504044$, Thermo Fisher Scientific Inc., MA, USA), $96 \mathrm{U} / \mathrm{mL}$ benzylpenicillin sodium, and $96 \mu \mathrm{g} / \mathrm{mL}$ streptomycin sulfate (\#15140122, Thermo Fisher Scientific Inc., MA, USA). All diluted in DMEM (Low glucose) (\#D6046, Merck \& Co., Inc., NJ, USA)

FGF receptor inhibitor (FGFRi) stock: PD 173074 (\#P2499-5MG, Merck \& Co., Inc., NJ, USA) diluted in DMSO (\#D2650-5X5ML, Merck \& Co., Inc., NJ, USA)

Rho-kinase inhibitor (Y) stock (8-10 mM): CultureSure Y-27632 (\#036-24023, FUJIFILM Wako Pure Chemical Corporation, Japan) diluted in distilled water (Otsuka Pharmaceutical Factory, Japan) to a final $10 \mu \mathrm{M}$ concentration when added to the cell culture medium

TGF-ß/Activin/Nodal signal inhibitor (SB) stock (4-5 $\mathrm{mM}$ ): SB 431542 hydrate (\#S4317-5MG, Merck \& Co., Inc., NJ, USA) diluted in DMSO (\#D2650-5X5ML, Merck \& Co., Inc., NJ, USA) to a final $5 \mu \mathrm{M}$ concentration when added to the cell culture medium

Wnt signal inhibitor (CK) stock (2.4-3 mM): CKI-7 dihydrochloride (\#C0742-5MG, Merck \& Co., Inc., NJ, USA) diluted in distilled water (Otsuka Pharmaceutical Factory, Japan) to a final $3 \mu \mathrm{M}$ concentration when added to the cell culture medium

RPE adhesion medium: DMEM/F12 (D8437, Merck \& Co., Inc., NJ, USA), 10\% FBS (12007C, Nichirei Corporation, Japan)

RPE washing solution: 98\% DMEM/F12 (D8437, Merck \& Co., Inc., NJ, USA), 1 mM Sodium pyruvate (S8636, Merck \& Co., Inc., NJ, USA), 2 mM L-Glutamine (G7513, Merck \& Co., Inc., NJ, USA)

\section{Labware}

For human use: micropipette tip, 2140-05-HR/2149P05/61849, Thermo Fisher Scientific Inc. (MA, USA); micropipette tip, 30389165, Mettler Toledo (OH, USA); micropipette tip, 737251, Greiner Bio-One International $\mathrm{GmbH}$ (Germany); disposable pipette, 356507, Corning Incorporated (NY, USA); disposable pipette, 
606160/607160/760160/768160, Greiner Bio-One International GmbH (Germany); filtration, SLGVJ13SL, Merck \& Co., Inc. (NJ, USA); filtration, SS-10LZ, Terumo Corporation (Japan); filtration, 431096/430281/431097/430282, Corning Incorporated (NY, USA); 1.5-mL tube, 72.692MS, Sarstedt K.K. (Japan); 15-mL tube, 352096, Corning Incorporated (NY, USA); 50-mL tube, 352070, Corning Incorporated (NY, USA).

For LabDroid use: 6-well plate, 353046, Corning Incorporated (NY, USA); $50 \mathrm{~mL}$ tube, MS-58500, Sumitomo Bakelite Co., Ltd. (Japan); micropipette tip, 3511-05-HR/3512-05-

HR/94410313/94410713/94052550, Thermo Fisher Scientific Inc. (MA, USA).

\section{LabDroid Maholo booth}

LabDroid including peripheral equipment were placed inside a booth made of acrylic walls and a stainless steel frame with three fan-filter-units. The LabDroid booth included a dual-arm humanoid (Robotic Biology Institute Inc., Japan), a $\mathrm{CO}_{2}$ incubator (APC-30D, ASTEC Co., Ltd., Japan), micropipettes (4641110N/4641030N/4641230N/4641210N, Thermo Fisher Scientific Inc., MA, USA), a tube rack (Robotic Biology Institute Inc., Japan), a plate rack (Robotic Biology Institute Inc., Japan), a dry bath (EC-40RA, AS ONE Corporation, Japan), a tip sensor (Robotic Biology Institute Inc., Japan), an aspirator (SP-30, Air Liquide, Italy), a dust bin (EPD3S, Sekisui Techno Molding Co., Ltd., Japan), and a microscope (EVOS FL Auto 2, Thermo Fisher Scientific Inc., MA, USA).

\section{hiPSC culture - initiation and preparation of cell suspensions (human part)}

The hiPSC line 253G1 (22), made from human dermal fibroblast, was obtained from RIKEN BRC (HPS0002). The hiPSCs were cultured and differentiated with the method described previously (23-25).

On DDay-14, frozen hiPSCs were initiated by the following procedures: first, laminin-coated 6-well plates were prepared. Final concentration of $0.5 \mu \mathrm{g} / \mathrm{cm}^{2}$ of iMatrix-511 (Matrixome Inc, Japan) diluted by PBS (-) was then added to each well of the four 6-well plates and incubated for a minimum of $60 \mathrm{~min}$ at $37^{\circ} \mathrm{C}$ and $5 \% \mathrm{CO}_{2}$, after which $0.75 \mathrm{~mL} /$ well of hiPSC maintenance medium was added. The supernatant was then removed. Next, 1 $\mathrm{mL} /$ well of hiPSC maintenance medium containing Rhokinase inhibitor (final $10 \mu \mathrm{M}$ concentration) was added and the coated plates were incubated at $37^{\circ} \mathrm{C}$ and $5 \%$ $\mathrm{CO}_{2}$ until further use.

For hiPSCs initiation, frozen vials of hiPSCs stored in liquid nitrogen were thawed in a water bath set at $37^{\circ} \mathrm{C}$ and suspended in $5 \mathrm{~mL}$ of hiPSC maintenance medium. After centrifugation $\left(160 \times \mathrm{g}, 22^{\circ} \mathrm{C}, 4 \mathrm{~min}\right)$, the supernatant was removed and an appropriate volume of hiPSC maintenance medium with a final $10 \mu \mathrm{M}$ Rhokinase inhibitor concentration was added. After counting the cells with a hemocytometer, the cells were seeded into laminin-coated 6-well plates at 43,300-45,000 cells $/ 1.5 \mathrm{~mL}$ medium/well.

On DDay -13 , the medium was replaced with hiPSC maintenance medium without Rho-kinase inhibitor. On DDays -12 to -8 , the medium was replaced with the same medium composition at 24-72 h intervals. On DDay -7, cells were collected from the plate, and cell suspensions were delivered to the LabDroid booth. The medium was aspirated, $2 \mathrm{~mL} /$ well of PBS (-) was gently added and then aspirated for washing, and then $1 \mathrm{~mL}$ of $0.5 \times$ TrypLE Select CTS (\#A12859-01, Thermo Fisher Scientific Inc., MA, USA) diluted in $0.5 \mathrm{mM}$ EDTAIPBS (-) was added, followed by incubation at $37^{\circ} \mathrm{C}$ and $5 \% \mathrm{CO}_{2}$ for $10-20 \mathrm{~min}$. Then, cells were detached by pipetting and collected into a $50-\mathrm{mL}$ tube, to which $1 \mathrm{~mL}$ of hiPSC maintenance medium and $3 \mathrm{~mL}$ of PBS (-) were added. After centrifugation $\left(160 \times g, 22^{\circ} \mathrm{C}, 4 \mathrm{~min}\right)$, the supernatant was removed, $0.75 \mathrm{~mL}$ of hiPSC maintenance medium with $10 \mu \mathrm{M}$ Rho-kinase inhibitor was added, and the cells were resuspended. The cell suspension was filtered through a 40- $\mu \mathrm{m}$ cell strainer (\#352340, Corning Incorporated, USA) with an additional $0.75 \mathrm{~mL}$ of hiPSC maintenance medium. After counting the cells with a hemocytometer, the cell suspension was set to 133,400 cells $/ 20 \mathrm{~mL}$ with hiPSC maintenance medium containing $10 \mu \mathrm{M}$ Rhokinase inhibitor in eight $50-\mathrm{mL}$ tubes. To prepare the cell suspensions, eight 6 -well plates coated with laminin were prepared. A final concentration of $0.5 \mu \mathrm{g} / \mathrm{cm}^{2}$ of iMatrix511 (Matrixome Inc., Japan) diluted in PBS (-) was added to each well of four 6-well plates and incubated for a minimum of $60 \mathrm{~min}$ at $37^{\circ} \mathrm{C}$ and $5 \% \mathrm{CO}_{2}$.

\section{iPSC-RPE differentiation (LabDroid part)}

On DDay -7 , the hiPSC suspension was seeded into eight 6 -well plates by coating eight 6 -well plates with laminin, and placing eight tubes of the iPSC suspension and labware in the appropriate positions. The task of seeding was initiated, and the robotic operation was performed by LabDroid (Figure $\mathbf{2 C}$ ). After the robotic operation, the eight cell-seeded plates were exported and incubated at a $\mathrm{CO}_{2}$ incubator outside the LabDroid booth.

On DDay -6 , the eight seeded plates were imported into the $\mathrm{CO}_{2}$ incubator of the LabDroid booth. The users prepared eight $50-\mathrm{mL}$ tubes of hiPSC maintenance medium with a final $10 \mu \mathrm{M}$ Rho-kinase inhibitor concentration and two 50-mL tubes of hiPSC maintenance medium with final $5 \mu \mathrm{M}$ FGFRi and $10 \mu \mathrm{M}$ Rho-kinase inhibitor concentrations. The reagents and labware were placed in the appropriate positions. The task of preconditioning was then initiated, and the robotic operation was performed by LabDroid (medium exchange type I; Figure 2D).

On DDays -5 to -1 , the users prepared eight $50-\mathrm{mL}$ tubes of hiPSC maintenance medium without Rho-kinase inhibitor and two $50-\mathrm{mL}$ tubes of hiPSC maintenance medium with a final $5 \mu \mathrm{M}$ FGFRi concentration. The reagents and labware were placed in the appropriate 
positions. The task of preconditioning was initiated, and the robotic operation was performed by LabDroid (medium exchange type I; Figure 2D).

On DDay 0, the following procedure was used for the operation of four plates: the users prepared four 6-well plates coated with laminin. A final $0.5 \mu \mathrm{gg} / \mathrm{cm}^{2}$ concentration of iMatrix-511 (Matrixome Inc., Japan) diluted in PBS (-) was added to each well of the four 6well plates and then the plates were incubated for a minimum of $60 \mathrm{~min}$ at $37^{\circ} \mathrm{C}$ and $5 \% \mathrm{CO}_{2}$. The users also prepared two $50-\mathrm{mL}$ tubes of PBS (-), two 50-mL tubes of $0.5 \times$ TrypLE Select CTS (\#A12859-01, Thermo Fisher Scientific Inc., MA, USA) diluted in 0.5 mM EDTA/PBS (-), and four plates with RPE differentiation medium $(20 \%$ KSR) with final $10 \mu \mathrm{M}$ Rho-kinase inhibitor/3 $\mu \mathrm{M}$ Wnt signal inhibitor/5 $\mu \mathrm{M}$ TGF- $\beta /$ Activin/Nodal signal inhibitor ( $4 \mathrm{~mL} /$ well each). The cell plates, laminin-coated plates, plates with medium, reagents, and labware were placed in the appropriate positions. The task of passage was initiated, and robotic operations were performed by LabDroid (Figure 2E). After performing this operation twice (four plates each), the eight cell-passaged plates were exported and incubated in a $\mathrm{CO}_{2}$ incubator outside the LabDroid booth.

On DDay 1, the eight cell-passaged plates were imported into the $\mathrm{CO}_{2}$ incubator of the LabDroid booth. Users prepared eight $50-\mathrm{mL}$ tubes of RPE differentiation medium ( $10 \% \mathrm{KSR}$ ), two $50-\mathrm{mL}$ tubes of $100 \% \mathrm{KSR}$, one $50-\mathrm{mL}$ tube of $4 \mathrm{mM}$ Rho-kinase inhibitor stock/1.2 mM Wnt signal inhibitor stock, and one $50-\mathrm{mL}$ tube of $4 \mathrm{mM}$ TGF- $\beta /$ Activin/Nodal signal inhibitor stock. The reagents and labware were placed in the appropriate positions. The task of RPE differentiation was initiated, and the robotic operation was performed by LabDroid (medium exchange type I; Figure 2D).

On DDays 2 to 19, the users prepared eight $50-\mathrm{mL}$ tubes of RPE differentiation medium (10\% KSR), two 50$\mathrm{mL}$ tubes of $100 \% \mathrm{KSR}$, one $50-\mathrm{mL}$ tube of $4 \mathrm{mM}$ Rhokinase inhibitor stock/1.2 mM Wnt signal inhibitor, and one $50-\mathrm{mL}$ tube of $4 \mathrm{mM}$ TGF-B/Activin/Nodal signal inhibitor. The reagents and labware were placed in the appropriate positions. The task of RPE differentiation was initiated, and the robotic operation was performed by LabDroid (medium exchange type I; Figure 2D).

On DDays 20 to 32, the users prepared eight $50-\mathrm{mL}$ tubes of RPE differentiation medium (10\% KSR). The reagents and labware were placed in the appropriate positions. RPE differentiation and maintenance were initiated and the robotic operations were performed by LabDroid (medium exchange type I; Figure 2F).

\section{Scoring - sampling}

On DDay 33, the cell plates were exported and the cell culture medium was replaced with fresh RPE maintenance medium. After $24 \mathrm{~h}$ (DDay 34), the medium was collected for ELISA analysis. The remaining media were aspirated, $2 \mathrm{~mL}$ of PBS (-) were added, and were then aspirated again for washing. After that, photographic images were acquired for the calculation of scoring values.

\section{Scoring - image analysis}

Images were acquired using a digital camera (PSG7X MARKII, Canon Inc., Japan): ISO 500; focal length $\mathrm{F}=9.00,50 \mathrm{~mm}$; exposure time, $1 / 1250 \mathrm{sec}$. The camera was set in the same position throughout all experiments. The acquired images were automatically processed by filtering with Gaussian blur, subtracting the background, binarizing by thresholding with a constant value, and cropping with a constant pixel value. The colored cell area was then calculated.

\section{Purification and storage}

Purification of iPSC-RPE cells was conducted using the same protocol described in a study previously reported (7). When the RPE colonies reached an appropriate size, the cells were suspended in RPE maintenance medium and kept as a floating culture for about 10 days in a low cell adhesion plate (MS-90600Z, Sumitomo Bakelite Co., Ltd., Japan). Under the microscope, colonies consisting only of black RPE cells were selected. Then, they were transferred to 12-well plates coated with iMatrix, and cultured in RPE adhesion medium/RPE maintenance medium (1:1). Once the RPE cell colonies became attached to the dish, they were cultured in RPE maintenance medium with basic fibroblast growth factor (bFGF), which was changed every 2-3 days.

After 10-12 days of cell selection, unsuitable cells were removed, and the cells were passaged. The medium was aspirated and $1 \mathrm{~mL}$ of RPE washing solution was added and aspirated again for washing. Then, 0.5 $\mathrm{mL}$ of RPE washing solution was added and atypical cells were eliminated using micropipette tips under microscope observation. After the removal process, the medium was aspirated, $1 \mathrm{~mL} /$ well of PBS (-) was added and aspirated for washing, and then $0.5 \mathrm{~mL}$ of Trypsin-EDTA solution (203-20251, FUJIFILM Wako Pure Chemical Corporation, Japan) was added, followed by incubation at RT (approximately $25^{\circ} \mathrm{C}$ ) and $5 \% \mathrm{CO}_{2}$ for 8-10 minutes. Cells were detached by pipetting and collected into a 50 $\mathrm{mL}$ tube. After centrifugation $\left(280 \times \mathrm{g}, 25^{\circ} \mathrm{C}, 4 \mathrm{~min}\right)$, the supernatant was removed, and the pellet was resuspended in $1 \mathrm{~mL} /$ plate of RPE adhesion medium/RPE maintenance medium (1:1) and filtered through a $40-\mu \mathrm{m}$ cell strainer (352340, Corning Incorporated, NY, U.S.A.). After counting the cells with a hemocytometer, the cells were seeded into 12-well plates. The medium was changed to RPE maintenance medium with bFGF.

After 1-3 days of cell passage, the medium was aspirated, the cells were washed with $0.5 \mathrm{~mL}$ of RPE maintenance medium, and $1 \mathrm{~mL}$ of RPE maintenance medium containing $10 \mathrm{ng} / \mathrm{mL}$ bFGF and $0.5 \mu \mathrm{M}$ SB431542 was added. This medium was exchanged every 2-3 days. 
The cells were stored when they formed hexagonal shapes after sufficient confluency. For that, the medium was aspirated, $1 \mathrm{~mL} /$ well of PBS (-) was added and then aspirated for washing, and $0.5 \mathrm{~mL}$ of Trypsin-EDTA solution (203-20251, FUJIFILM Wako Pure Chemical Corporation, Japan) was added, followed by incubation at $37{ }^{\circ} \mathrm{C}$ and $5 \% \mathrm{CO}_{2}$ for $10-15$ minutes. After adding $>0.5$ $\mathrm{mL}$ of RPE adhesion medium, the cells were detached using a cell scraper (MS-93100, Sumitomo Bakelite Co., Ltd., Japan). The cell suspension was filtered through a 40- $\mu \mathrm{m}$ cell strainer (352340, Corning Incorporated, NY, USA) and then centrifuged for $4 \mathrm{~min}$ at $280 \times g$ to obtain a cell pellet. The pellet was resuspended in $1 \mathrm{~mL}$ of RPE adhesion medium/RPE maintenance medium (1:1) and filtered through a 40- $\mu \mathrm{m}$ cell strainer. After counting the cells with a hemocytometer, the cell suspension was centrifuged for $4 \mathrm{~min}$ at $280 \times g$ to obtain a cell pellet. Then, STEM-CELLBANKER (CB047, Zenoaq Resource Co., Ltd., Japan) was added until a cell concentration of 500,000 cells $/ 0.5 \mathrm{~mL} / \mathrm{tube}$, and the cell suspensions were dispensed into cryovials. The cryotubes were placed in a cell freezing container at $-80^{\circ} \mathrm{C}$ for $3-24 \mathrm{~h}$, and then stored at $-150^{\circ} \mathrm{C}$.

\section{Initiation of iPSC-RPE stock and recovery culture}

Frozen vials of RPE cells were thawed in a $37^{\circ} \mathrm{C}$ water bath and suspended in $4.5 \mathrm{~mL}$ of RPE adhesion medium. After centrifugation $\left(280 \times g, 25^{\circ} \mathrm{C}, 4 \mathrm{~min}\right)$, the supernatant was removed and RPE adhesion medium/RPE maintenance medium (1:1) was added. After counting the cells with a hemocytometer, the cells were seeded into 24 -well plates $(0.5 \mathrm{~mL} /$ well $)$.

After 1-3 days of cell seeding, the medium was aspirated, the cells were washed with $0.25 \mathrm{~mL}$ of RPE maintenance medium, and $0.5 \mathrm{~mL} /$ well of RPE maintenance medium containing $10 \mathrm{ng} / \mathrm{mL}$ bFGF and 0.5 $\mu \mathrm{M}$ SB431542 was added. This same type of medium was exchanged every $2-3$ days.

Two weeks after seeding, the RPE cells were passaged. Two weeks after cell passage, the RPE cells were used for cell biological validation processes (RTPCR, ELISA, and immunohistochemistry).

\section{Validation - RT-PCR}

Total RNA was extracted from transfected cells using RNeasy Micro Kit (\#74004, QIAGEN, Germany). Firststrand cDNA synthesis was performed on 500-1000 ng of total RNA, using SuperScript III (\#18080-044, Thermo Fisher Scientific Inc., MA, USA) according to the manufacturer's instructions. Each mRNA transcript was amplified using PCR with the following primers:

BEST1 (+), 5'-dTAGAACCATCAGCGCCGTC

BEST1 (-), 5'-dTGAGTGTAGTGTGTATGTTGG

RPE65 (+), 5'-dTCCCCAATACAACTGCCACT

RPE65 (-), 5'-dCCTTGGCATTCAGAATCAGG

CRALBP (+), 5'-dGAGGGTGCAAGAGAAGGACA

CRALBP (-), 5'-dTGCAGAAGCCATTGATTTGA

GAPDH (+), 5'-dACCACAGTCCATGCCATCAC

\section{GAPDH (-), 5'-dTCCACCACCCTGTTGCTGTA}

\section{Validation - ELISA}

The collected media were centrifuged $\left(90 \times g, 4^{\circ} \mathrm{C}, 1\right.$ $\min$ ), and the supernatant was collected and stored at $-80^{\circ} \mathrm{C}$. The amount of VEGF contained in the thawed medium was measured using the protocols and reagents from the VEGF Human ELISA Kit (BMS277-2, Thermo Fisher Scientific, USA), and the amounts of PEDF were measured using a Human ELISA Kit (RD191114200R, BioVendor, Czech Republic).

\section{Validation - immunohistochemistry}

Cells were washed with PBS (-), fixed in $15 \%$ paraformaldehyde for $1 \mathrm{~h}$ at RT (approximately $25^{\circ} \mathrm{C}$ ), and stored at $4^{\circ} \mathrm{C}$ after removal of PFA and addition of PBS (-). After removal of the solutions, cells were treated with $50 \mu \mathrm{L} /$ well of $0.2 \%$ Triton X-100/PBS (-), incubated for 30 min at RT, washed with PBS (-), blocked with 50 $\mu \mathrm{L}$ of Blocking One (03953-95, Nacalai Tesque Inc., Japan), and incubated for $1 \mathrm{~h}$ at RT. After removal of the solutions, cells were stained at $4^{\circ} \mathrm{C}$ o/n in $50 \mu \mathrm{L}$ of the 1 st antibody diluent (rabbit anti-ZO-1, 61-7300, Thermo Fisher Scientific Inc., MA, USA; anti-MITF, mouse antiMiTF, ab80651, Abcam plc., Britain; antibody diluent, S2022, Agilent Technologies Inc., USA). After removal of the solutions, cells were washed with PBS (-) and then stained at RT for $1 \mathrm{~h}$ in $50 \mu \mathrm{L}$ of the $2 \mathrm{nd}$ antibody diluent (Alexa Fluor 546 Goat Anti-mouse IgG, A-11030, Thermo Fisher Scientific Inc., MA, USA; Alexa Fluor 488 Goat Anti-rabbit IgG, A-11034, Thermo Fisher Scientific Inc., MA, USA; antibody diluent, S2022, Agilent Technologies Inc., USA) with DAPI (1 $\mathrm{gg} / \mathrm{mL}$, D1206, Thermo Fisher Scientific Inc., MA, USA). After removal of the solutions, cells were washed with PBS (-), and then $50 \mu \mathrm{L}$ of PBS (-) was added. Images of immunohistochemistry samples were acquired using an IX73 inverted microscope (Olympus, Japan).

\section{Bayesian optimization module}

When no prior experimental results exist, the Bayesian optimization module generates the next query from random uniform sampling. When past experimental results are available, the Bayesian optimization module generates queries using two components: the Model updater and the Query generator (Figure 5A).

The Model updater updates the surrogate model to predict the experimental results given past experimental results: $D=\left\{\left(\boldsymbol{x}_{i}, y_{i}\right)\right\}_{i=1}^{n}$. We adopted Gaussian process regression (GPR) with the ARD-RBF kernel as the surrogate model to estimate the expected score and confidence level for all unevaluated experimental parameters. Based on the experimental results shown in Figure $3 \mathbf{C}$, the observation noise was assumed to follow a zero-mean Gaussian noise with a variance of 0.0039 at all points in the search space. By using the surrogate model, the Query generator generates the next queries in two steps. In step 1, the Query generator constructs an 
acquisition function that estimates the expected progress toward the optimal experimental parameter at a given experimental parameter $\boldsymbol{x}$ in the search space. We adopted the Expected improvement (EI) (26), a commonly used acquisition function in $\mathrm{BO}$. El estimates how much improvement over the current best score is expected from each point in the search space. In step 2, by using the acquisition function, the Query generator decides where to evaluate next, and our problem required the simultaneous performance of 48 experiments corresponding to 8 plates $\times 6$ wells in each round. In addition, because the trypsin processing time $(D P)$ is a batch contextual parameter as described herein, a policy function that generates parameter sets taking such structural context into account must be incorporated. Therefore, we developed the Batch Contextual Local Penalization (BCLP) as a policy function to generate multiple points with context in parallel. The BCLP is a batch generation policy that extends the local penalization (27) to be applied to cases where complex structural context parameters exist. As shown in Figure $5 \mathrm{C}$, for each value of the contextual parameter $D P$ in ascending order, BCLP iteratively generated the parameter by maximizing and penalizing the acquisition function 48 times to obtain the next experimental parameters $\boldsymbol{X}_{\text {next }}$ for each subsequent well. In addition, after each round, the more promising KP intervals were reconfigured by calculating the integral value of the acquisition function. We also replaced the queries that corresponded to the place of the top two pigmented scores in the previous experiments with the parameter of the top two pigmented scores in the previous experiments as a positive control.

\section{Statistical analysis}

Statistical analyzes were performed by Wolfram Mathematica version 11.2.0.0. In this study, $P<0.05$ was considered significant $\left({ }^{*} P<0.05,{ }^{* *} P<0.01,{ }^{* * *} P<0.001\right.$, and n.s. = not significant $)$.

\section{ACKNOWLEDGEMENTS}

We thank T. Mitsuyama, T. Iwata, and N. Yachie for their insightful comments; E. Takagi and $\mathrm{H}$. Hirabayashi for research support; $\mathrm{H}$. Uchida for illustrations; and J. Freeman for carefully proofreading the manuscript. We also thank all the laboratory members at RIKEN BDR, in particular, N. Koide, Y. Shibata, A. Maeda, and T. Maeda for their kind help in the preparation of the materials, their support in the experiments, and their insightful discussions. This work was supported by a grant from AMED (JP20bm0204002, AMED, to M. Takahashi), a project subsidized by the New Energy and Industrial Technology Development Organization (NEDO, to $\mathrm{M}$. Takahashi), JST-Mirai Program (JPMJMI18G4, JST, to K. T.), an internal grant from the RIKEN Center for Biosystems Dynamics Research (RIKEN, to $M$. Takahashi and K. T.), RIKEN Engineering Network
(RIKEN, to M. Takahashi), RIKEN Junior Research Associate program for graduate students (RIKEN, to N. M.).

\section{AUTHOR CONTRIBUTIONS}

K.T., and T.N. conceived of the presented idea. G.N.K., T.T., T.S., Y.O., M. Takahashi, and T.N. designed the project. G.N.K., M. Terada, N.S., N.M., T.M., M.N., and G.A.S. performed the biological experiments. T.T., C.T.W., T.H., S.A.H., T.S., and Y.O. performed software development and informatics experiments. T.K., M.K., K.M., and T.N. performed robot teaching and maintenance. T.S. performed the project management. G.N.K performed the project administration. M. Takahashi, and T.N. supervised the project. G.N.K., T.T., and Y.O. wrote the manuscript. All authors discussed the results and commented on the manuscript.

\section{DECLARATION OF INTERESTS}

G.N.K., T.K., M.K., K.M., and T.N. are employees, executives, or stakeholders of Robotic Biology Institute Inc., which may benefit financially from the increased scientific use of LabDroid Maholo. T.T., C.T.W., T.H., S.A.H., T.S., Y.O., and K.T. are employees, shareholders, or stakeholders of Epistra Inc., which may benefit financially from the increased scientific use of developed software. All other authors declare no competing interests.

\section{REFERENCES}

1. H. Kitano, Artificial Intelligence to Win the Nobel Prize and Beyond: Creating the Engine for Scientific Discovery. AlMag. 37, 39 (2016).

2. R. D. King, J. Rowland, S. G. Oliver, M. Young, W. Aubrey, E. Byrne, M. Liakata, M. Markham, P. Pir, L. N. Soldatova, A. Sparkes, K. E. Whelan, A. Clare, The automation of science. Science. 324, 85-89 (2009).

3. P. Nikolaev, D. Hooper, F. Webber, R. Rao, K. Decker, M. Krein, J. Poleski, R. Barto, B. Maruyama, Autonomy in materials research: a case study in carbon nanotube growth. npj Computational Materials. 2, 16031 (2016).

4. P. B. Wigley, P. J. Everitt, A. van den Hengel, J. W. Bastian, M. A. Sooriyabandara, G. D. McDonald, K. S. Hardman, C. D. Quinlivan, P. Manju, C. C. N. Kuhn, I. R. Petersen, A. N. Luiten, J. J. Hope, N. P. Robins, M. R. Hush, Fast machine-learning online optimization of ultra-cold-atom experiments. Sci. Rep. 6, 25890 (2016).

5. M. HamediRad, R. Chao, S. Weisberg, J. Lian, S. Sinha, H. Zhao, Towards a fully automated algorithm driven platform for biosystems design. Nat. Commun. 10, 5150 (2019).

6. M. Kino-oka, Y. Sakai, Preface of the special issue "Cell Manufacturability." Regenerative Therapy. 12, 
1 (2019).

7. M. Mandai, A. Watanabe, Y. Kurimoto, Y. Hirami, C. Morinaga, T. Daimon, M. Fujihara, H. Akimaru, N. Sakai, Y. Shibata, M. Terada, Y. Nomiya, S. Tanishima, M. Nakamura, H. Kamao, S. Sugita, A. Onishi, T. Ito, K. Fujita, S. Kawamata, M. J. Go, C. Shinohara, K.-I. Hata, M. Sawada, M. Yamamoto, S. Ohta, Y. Ohara, K. Yoshida, J. Kuwahara, Y. Kitano, N. Amano, M. Umekage, F. Kitaoka, A. Tanaka, C. Okada, N. Takasu, S. Ogawa, S. Yamanaka, M. Takahashi, Autologous Induced Stem-Cell-Derived Retinal Cells for Macular Degeneration. N. Engl. J. Med. 376, 1038-1046 (2017).

8. N. Yachie, Robotic Biology Consortium, T. Natsume, Robotic crowd biology with Maholo LabDroids. Nat. Biotechnol. 35, 310-312 (2017).

9. B. Burger, P. M. Maffettone, V. V. Gusev, C. M. Aitchison, Y. Bai, X. Wang, X. Li, B. M. Alston, B. Li, R. Clowes, N. Rankin, B. Harris, R. S. Sprick, A. I. Cooper, A mobile robotic chemist. Nature. 583, 237-241 (2020).

10. A. E. Gongora, B. Xu, W. Perry, C. Okoye, P. Riley, K. G. Reyes, E. F. Morgan, K. A. Brown, A Bayesian experimental autonomous researcher for mechanical design. Sci Adv. 6, eaaz1708 (2020).

11. M. Kino-Oka, S. R. Chowdhury, Y. Muneyuki, M. Manabe, A. Saito, Y. Sawa, M. Taya, Automating the expansion process of human skeletal muscle myoblasts with suppression of myotube formation. Tissue Eng. Part C Methods. 15, 717-728 (2009).

12. A. Nishimura, R. Nakajima, R. Takagi, G. Zhou, D. Suzuki, M. Kiyama, T. Nozaki, T. Owaki, T. Takahara, S. Nagai, T. Nakamura, M. Sugaya, K. Terada, Y. Igarashi, H. Hanzawa, T. Okano, T. Shimizu, M. Yamato, S. Takeda, Fabrication of tissue-engineered cell sheets by automated cell culture equipment. J. Tissue Eng. Regen. Med. 13, 2246-2255 (2019).

13. F. F. dos Santos, P. Z. Andrade, C. L. da Silva, J. M. S. Cabral, Bioreactor design for clinical-grade expansion of stem cells. Biotechnol. J. 8, 644-654 (2013).

14. S. Konagaya, T. Ando, T. Yamauchi, H. Suemori, H. Iwata, Long-term maintenance of human induced pluripotent stem cells by automated cell culture system. Sci. Rep. 5, 16647 (2015).

15. R. J. Thomas, A. Chandra, P. C. Hourd, D. J. Williams, Cell Culture Automation and Quality Engineering: A Necessary Partnership to Develop Optimized Manufacturing Processes for Cell-Based Therapies. JALA: Journal of the Association for Laboratory Automation. 13, 152-158 (2008).

16. Y. Liu, P. Hourd, A. Chandra, D. J. Williams, Human cell culture process capability: a comparison of manual and automated production. J. Tissue Eng. Regen. Med. 4, 45-54 (2010).
17. F. A. C. Soares, A. Chandra, R. J. Thomas, R. A. Pedersen, L. Vallier, D. J. Williams, Investigating the feasibility of scale up and automation of human induced pluripotent stem cells cultured in aggregates in feeder free conditions. J. Biotechnol. 173, 53-58 (2014).

18. E. Matsumoto, N. Koide, H. Hanzawa, M. Kiyama, M. Ohta, J. Kuwabara, S. Takeda, M. Takahashi, Fabricating retinal pigment epithelial cell sheets derived from human induced pluripotent stem cells in an automated closed culture system for regenerative medicine. PLoS One. 14, e0212369 (2019).

19. H. Kitano, Computational systems biology. Nature. 420, 206-210 (2002).

20. M. Kino-oka, M. Mizutani, N. Medcalf, Cell manufacturability. Cell Gene Therapy Insights. 5, 1347-1359 (2019).

21. Y. Zhang, D. W. Apley, W. Chen, Bayesian Optimization for Materials Design with Mixed Quantitative and Qualitative Variables. Sci. Rep. 10, 4924 (2020).

22. M. Nakagawa, M. Koyanagi, K. Tanabe, K. Takahashi, T. Ichisaka, T. Aoi, K. Okita, Y. Mochiduki, N. Takizawa, S. Yamanaka, Generation of induced pluripotent stem cells without Myc from mouse and human fibroblasts. Nat. Biotechnol. 26, 101-106 (2008).

23. M. Haruta, Y. Sasai, H. Kawasaki, K. Amemiya, S. Ooto, M. Kitada, H. Suemori, N. Nakatsuji, C. Ide, Y. Honda, M. Takahashi, In vitro and in vivo characterization of pigment epithelial cells differentiated from primate embryonic stem cells. Invest. Ophthalmol. Vis. Sci. 45, 1020-1025 (2004).

24. H. Kawasaki, H. Suemori, K. Mizuseki, K. Watanabe, F. Urano, H. Ichinose, M. Haruta, M. Takahashi, K. Yoshikawa, S.-I. Nishikawa, N. Nakatsuji, Y. Sasai, Generation of dopaminergic neurons and pigmented epithelia from primate ES cells by stromal cell-derived inducing activity. Proc. Natl. Acad. Sci. U. S. A. 99, 1580-1585 (2002).

25. F. Osakada, H. Ikeda, M. Mandai, T. Wataya, K. Watanabe, N. Yoshimura, A. Akaike, Y. Sasai, M. Takahashi, Toward the generation of rod and cone photoreceptors from mouse, monkey and human embryonic stem cells. Nat. Biotechnol. 26, 215-224 (2008).

26. D. R. Jones, M. Schonlau, W. J. Welch, Efficient Global Optimization of Expensive Black-Box Functions. J. Global Optimiz. 13, 455-492 (1998).

27. J. Gonzalez, Z. Dai, P. Hennig, N. Lawrence, in Artificial Intelligence and Statistics (2016), pp. 648657. 
bioRxiv preprint doi: https://doi.org/10.1101/2020.11.25.392936; this version posted November 27, 2020. The copyright holder for this

preprint (which was not certified by peer review) is the author/funder. All rights reserved. No reuse allowed without permission.

\section{TABLE}

Table 1. Definition of optimized parameters

Parameter names, parameter name codes, description, parameter ranges, parameter units, correspondence between experimental procedure and parameters (related to Figures 2A, 4).

\begin{tabular}{|c|c|c|c|c|c|}
\hline Parameter name & Code & Description & Range & Unit & Prptocol step \\
\hline Preconditioning concentration & $P C$ & FGFRi concentration in medium & $10-505$ & $\mathrm{nM}$ & Preconditioning \\
\hline Preconditioning period & $P P$ & FGFRi duration in medium & $1-6$ & day & Preconditioning \\
\hline Detachment trypsin period & $D P$ & $\begin{array}{l}\text { Trypsin incubation duration at room temperature after } \\
\text { incubation at } 37^{\circ} \mathrm{C}, 20 \mathrm{~min} \text {. }\end{array}$ & $5,8,11,14,17,20,23$ & $\min$ & Passage \\
\hline Detachment pipetting strength & $D S$ & Pipetting strength during cell detachment & $10-100$ & $\mathrm{~mm} / \mathrm{sec}$ & Passage \\
\hline Detachment pipetting length & $D L$ & Bottom surface area to be pipetted & short/long & $\mathrm{N} / \mathrm{A}$ & Passage \\
\hline Three supplements period & $3 P$ & Three chemical supplements duration & $3-19$ & day & RPE differentiation \\
\hline
\end{tabular}

\section{FIGURES}

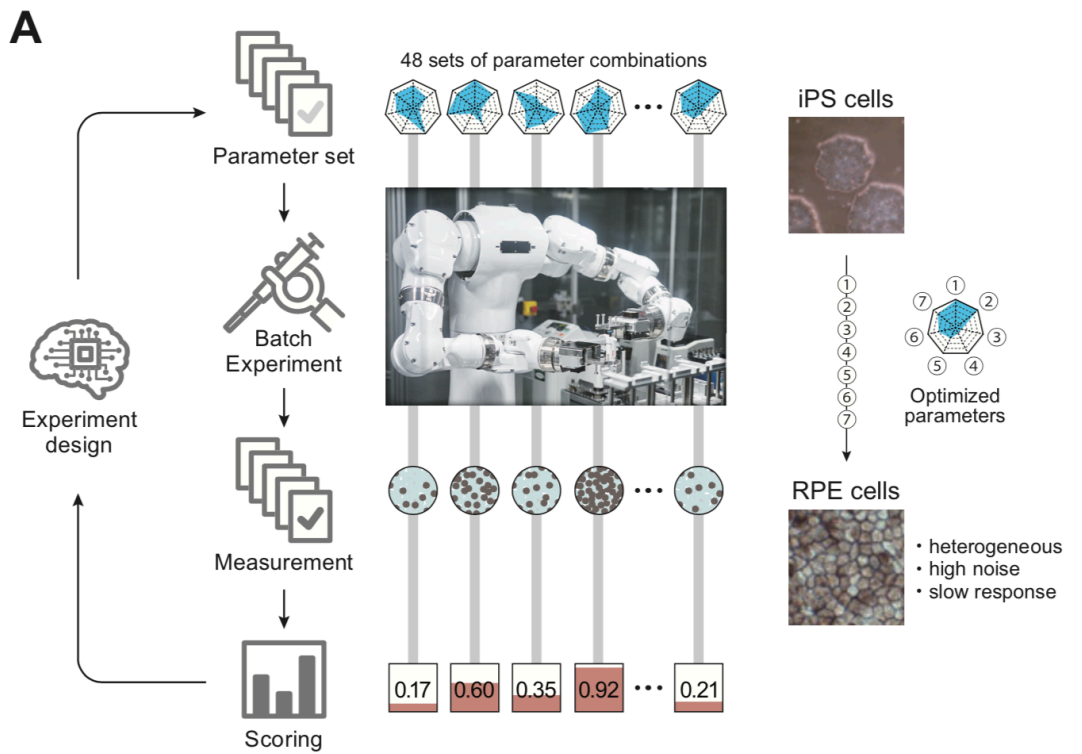

B

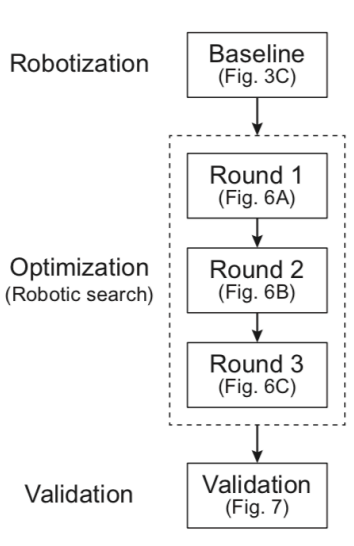

Figure 1. Robotic search for optimal experimental conditions

A, Overall workflow for the optimization of experimental procedures using combined experimental robotics and Bayesian optimization. The user defines the target experimental protocol, subject parameters in the protocol, and the validation function. In this study, we chose the differentiation procedure from iPS to RPE cells as a target protocol, and selected reagent concentration, administration period, and five other parameters (details are shown in Table 1). We defined the pigmented area in a culture well, which represents the degree of RPE differentiation induction, as the validation function. The optimization program presents a plurality of parameter candidates, LabDroid performs the experiment, and then an evaluation value for each candidate is obtained. Subsequently, the Bayesian optimization presents a plurality of parameter candidates predicted to produce higher validation values. The optimal parameters are searched by repeating candidate presentation, experiment execution, validation, and prediction.

B, Workflows performed in this study. First, robotization of the iPSC-RPE protocol was performed as a baseline. Next, the optimization process was conducted in three rounds, followed by statistical and biological validations. The figure numbers in parentheses represents the results shown in the figure. 


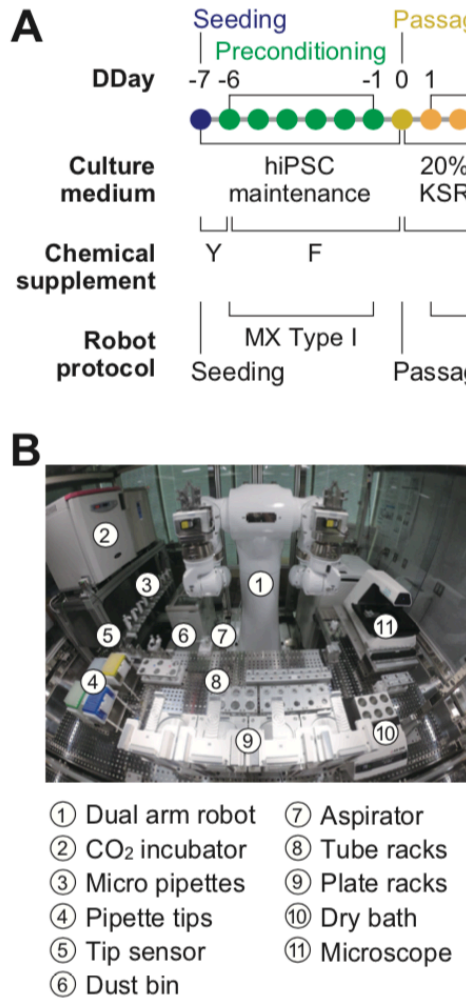

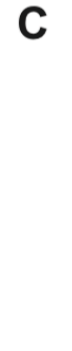

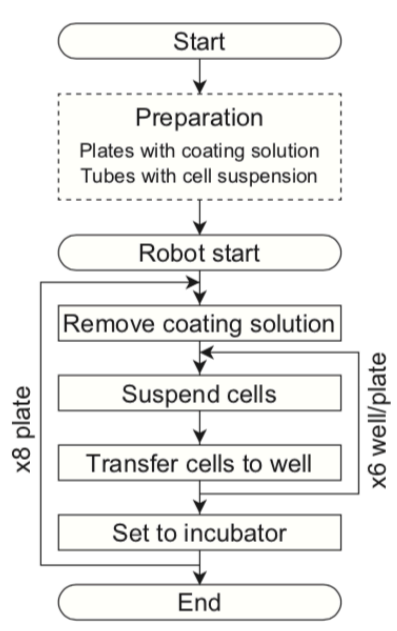

E

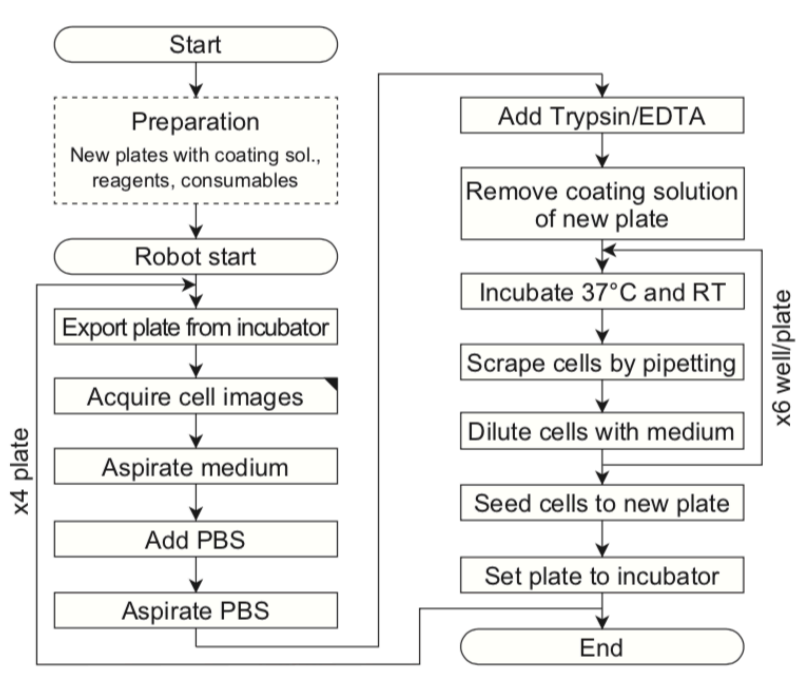

D

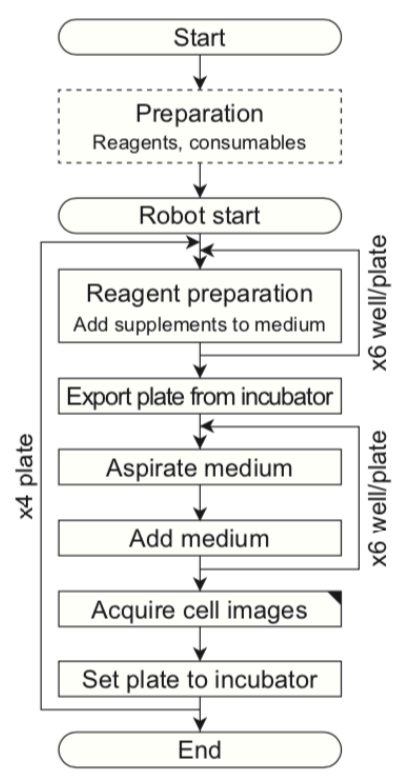

$\mathbf{F}$

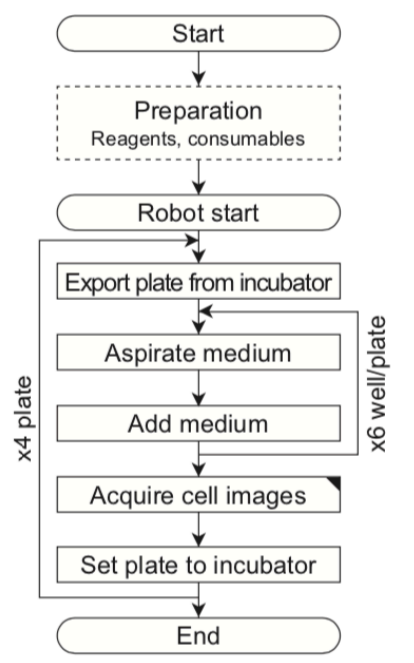

Figure 2. Robotization of iPSC-RPE differentiation protocols

A, Schematic diagram of the standard iPSC-RPE differentiation procedures. DDay indicates the differentiation day. Filled circles represent days when the robot operated, solid circles represent days with human operations only, and dashed line circles represent days when no operations were conducted. F stands for FGF receptor inhibitor; Y for Y-27632, a Rho-kinase inhibitor; SB for SB431542, a TGF- $\beta /$ Activin/Nodal signal inhibitor; CK for a CKI-7, Wnt signal inhibitor; and MX for medium exchange.

B, LabDroid Maholo including peripheral equipment.

C-F, Workflows of the experimental operation on seeding (C), medium exchange type I for preconditioning and the first part of differentiation induction (D), passage (E), medium exchange type II for the second part of differentiation induction, and RPE maintenance culture $(\mathbf{F})$. Dashed line rectangles represent the operations carried out by humans, and solid line rectangles represent the operations carried out by the robot. 
A

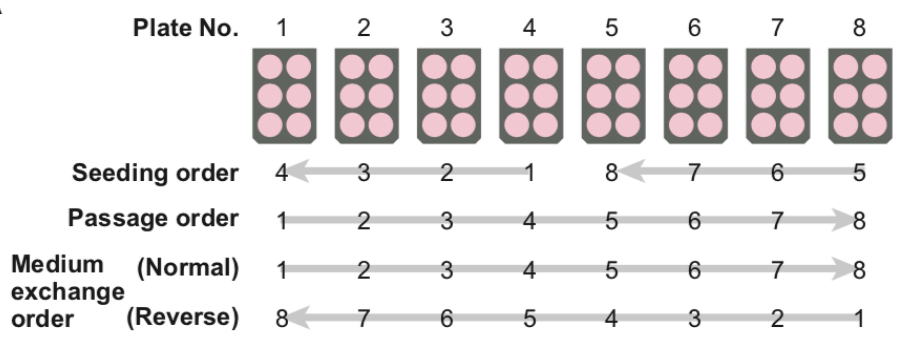

B

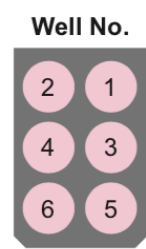

C

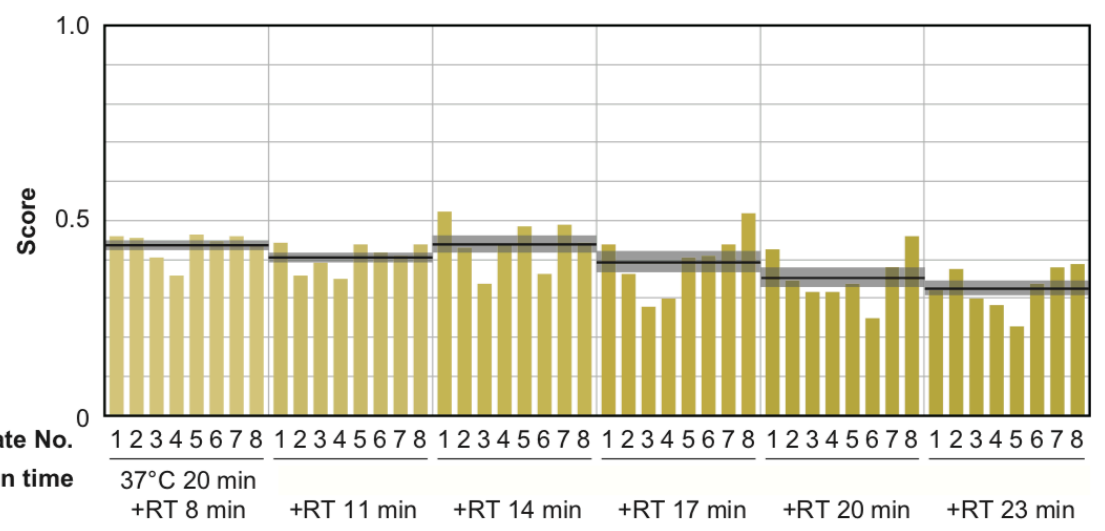

D

Plate No. 1

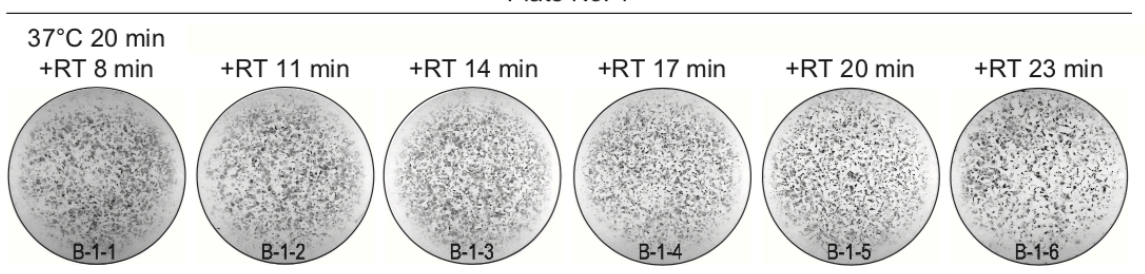

E

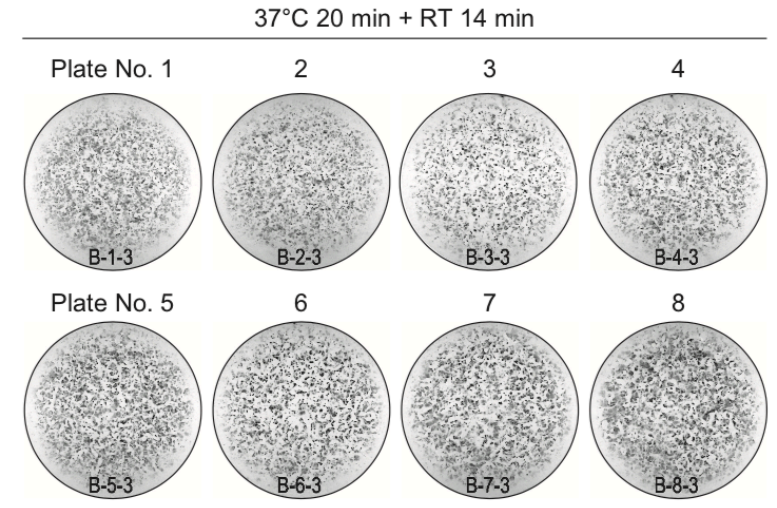

Figure 3. Execution of iPSC-RPE differentiation protocols

A, Plate numbering and the orders of seeding, passage, and medium exchange operations. Eight 6 -well plates were used for each experiment.

B, Well numbering.

C, Scores of the first trial. iPSC-RPE differentiation was conducted under six different trypsin treatment times using LabDroid. Vertical blue bars represent the pigmented cell area score of each well. The bold black lines and the shaded area around the lines represent the mean score and SEM of eight samples operated at the same trypsin time, respectively.

D-E, Representative pigmented images. Images acquired on DDay 34 in the baseline experiment of plate 1 (D) and of trypsin treatment for $20 \mathrm{~min}$ at $37^{\circ} \mathrm{C}+14 \mathrm{~min}$ at $\mathrm{RT}(\mathrm{E})$. ID labelling on the bottom reads 'B (baseline) - Plate No. - Well No.'. 
A

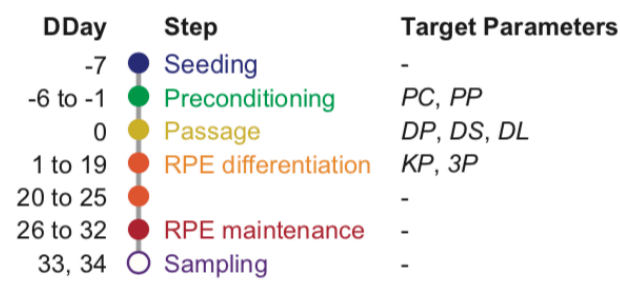

B

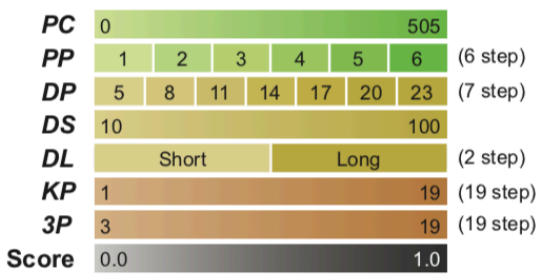

Figure 4. Parameterization of protocol

A, Definition of target parameters and corresponding steps in the protocol: $P C$, preconditioning concentration; $P P$, preconditioning period; $D P$, detachment trypsin period; $D S$, detachment pipetting strength; $D L$, detachment pipetting length; $K P$, KSR concentration reducing period; and $3 P$; three chemical $(\mathrm{Y}, \mathrm{SB}, \mathrm{CKI})$ supplement administration period.

B, Ranges and stepping of the parameters.

A

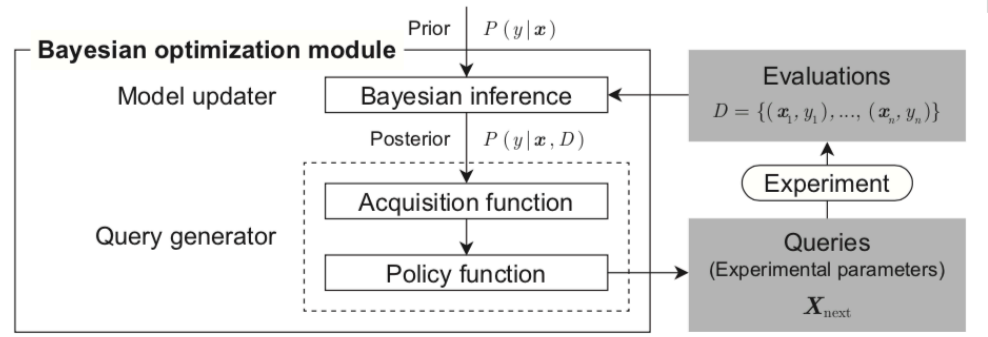

B

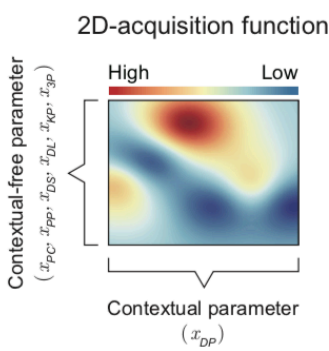

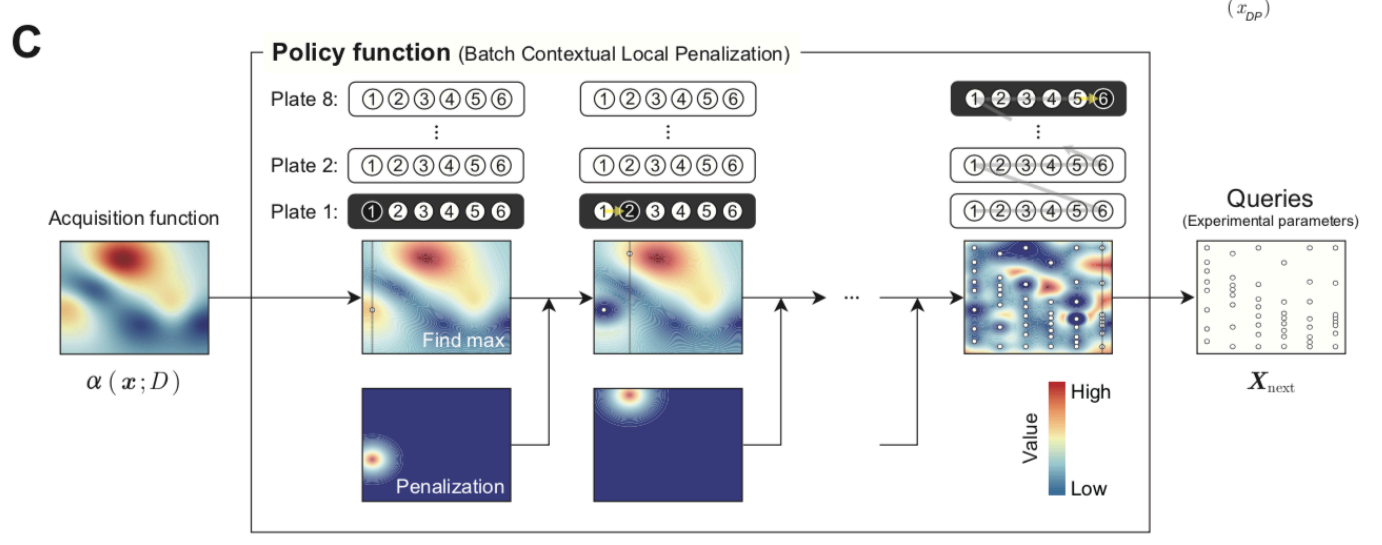

Figure 5. Bayesian optimization module

A, Bayesian optimization module consists of two components: Model updater, and Query generator. The Model updater updates the Gaussian process posterior on the experiment using all available data $D=\left\{\left(\boldsymbol{x}_{i}, y_{i}\right)\right\}_{i=1}^{n}$. The Query generator calculates the acquisition function $\alpha(\boldsymbol{x} ; D)$ for an experiment parameter $\boldsymbol{x}$ with the posterior distribution $P(y \mid \boldsymbol{x}, D)$, and generates the experiment parameter set $\boldsymbol{X}_{\text {next }}$ for the next 48 points using the policy function with $\alpha(\boldsymbol{x} ; D)$.

B-C, Test of the query generation process using a two-dimensional toy acquisition function.

B, Values of the toy acquisition function given an experimental parameter set. The horizontal axis represents input values of $x_{D P}$ (contextual parameter), and the vertical axis represents input values of the other six remaining contextual-free parameters $\boldsymbol{X}=\left(x_{P C}, x_{P P}, x_{D S}, x_{D L}, x_{3 P}, x_{K P}\right)$, which are collapsed into a single axis. The color of the heatmap shows the value of the acquisition function. In the heat map, the acquisition value is higher in places where the color is closer to red and lower in places where the color is closer to blue.

C, Test of the query generation process for the experimental parameter set $\boldsymbol{X}_{\text {next }}$ in the next experiment using Batch Contextual Local Penalization policy (BCLP policy). The heat maps in the upper row show the (penalized) acquisition function values, and lower row shows penalization values to the acquisition function. The Queries $\boldsymbol{X}_{\text {next }}$ for 48 wells (right side figure) are iteratively generated from the maximization-penalization loop on the acquisition function. 
A

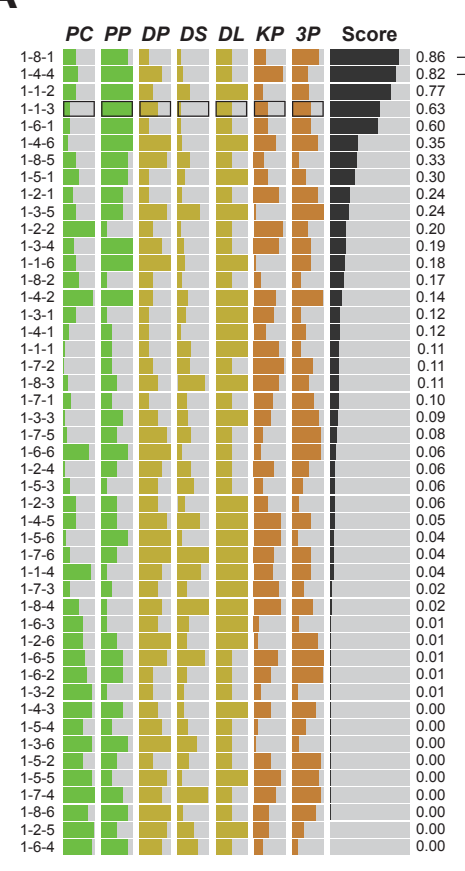

D

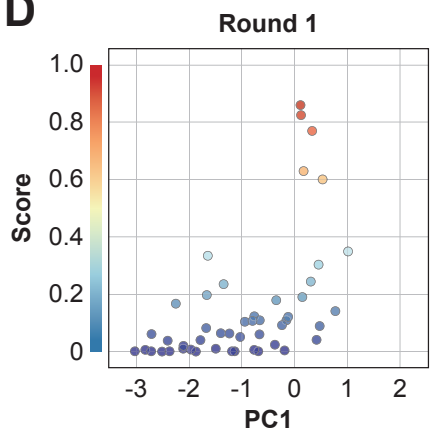

B PC PP DP DS DL KP $3 P$ Score
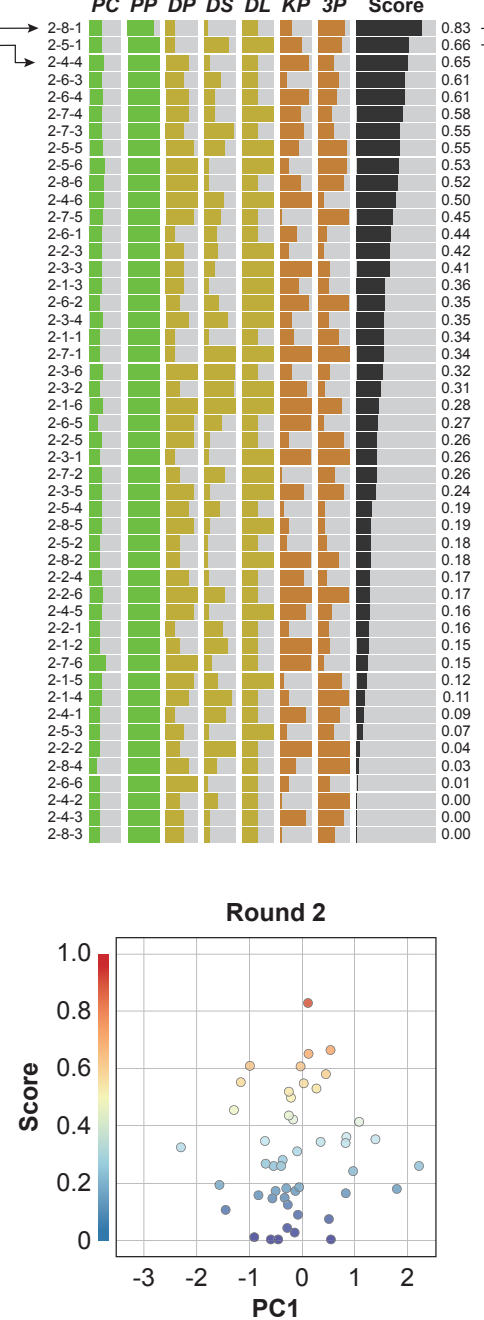

C
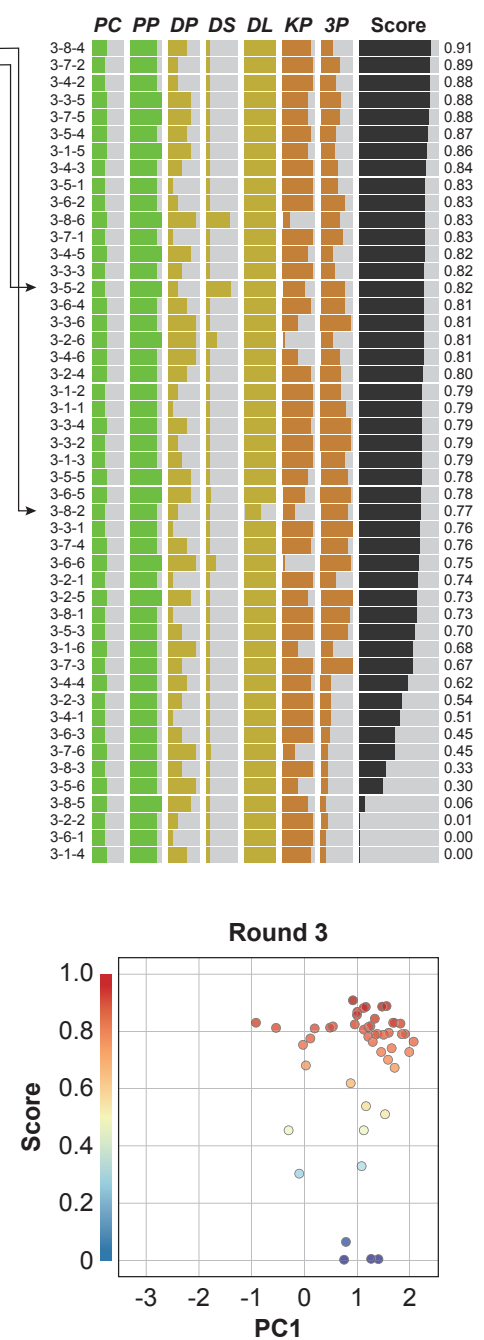

Figure 6. Automatic search for optimal parameters in iPSC-RPE differentiation

A-C, Parameter candidates sorted in order of the pigmentation score in optimization round 1 (A), 2 (B), and $3(\mathbf{C})$. The ID labelling on the left represents 'Round No. - Plate No. - Well No.'. For example, "1-2-3" means "(Round) 1-(Plate) 2-(Well) 3". Parameter values and resulting pigmentation scores are plotted as horizontal bars. The parameter candidate with black frames (1-1-3) in (A) is the standard condition. Arrows represent control experiments: the top 2 conditions in round 1 were included in round 2 , and the top 2 conditions in round 2 were implemented in round 3.

D, Visualization of the parameter set and pigmentation score distributions using partial least squares regression (PLS) in each round. The horizontal axis shows the value of the parameter candidates that are projected onto the first component of PLS. The vertical axis shows the pigmentation score of each parameter candidate. As rounds progress, the overall score tends to converge in a higher direction. 
A

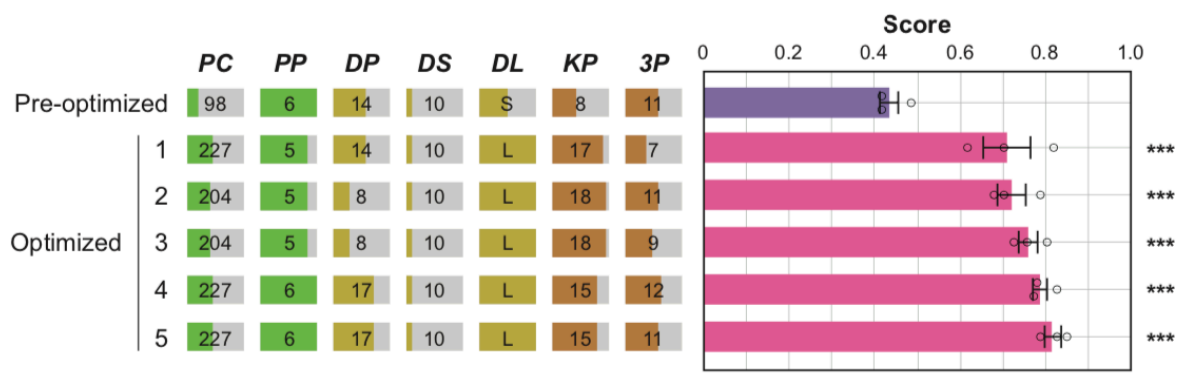

B

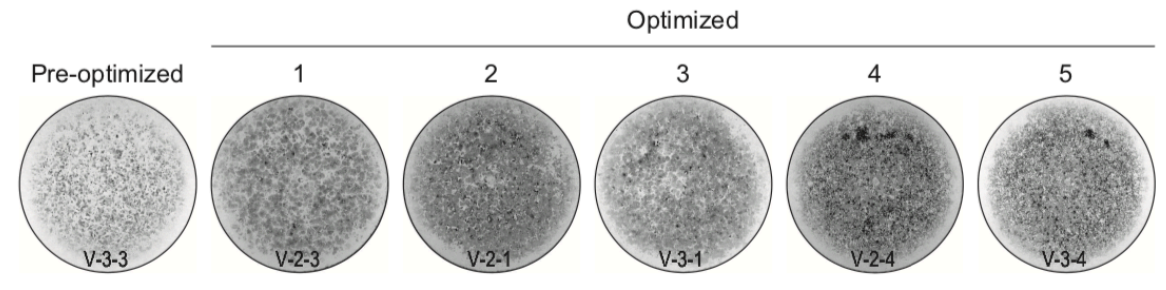

C

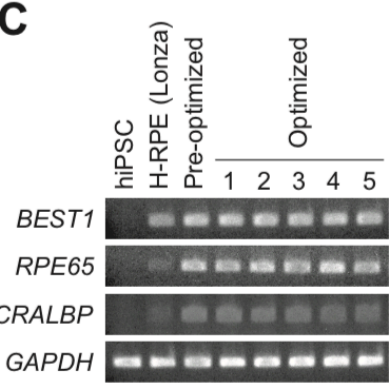

$\mathbf{F}$
D

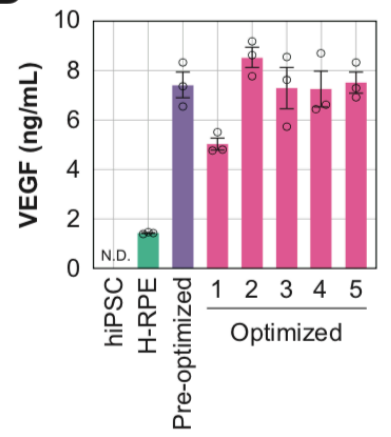

E

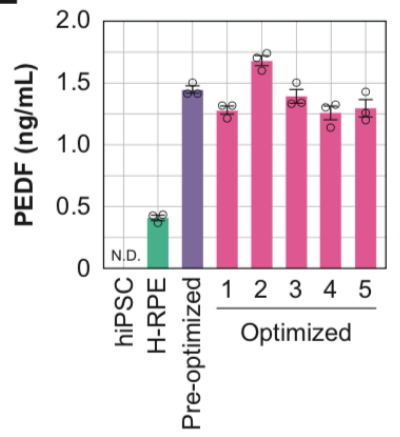

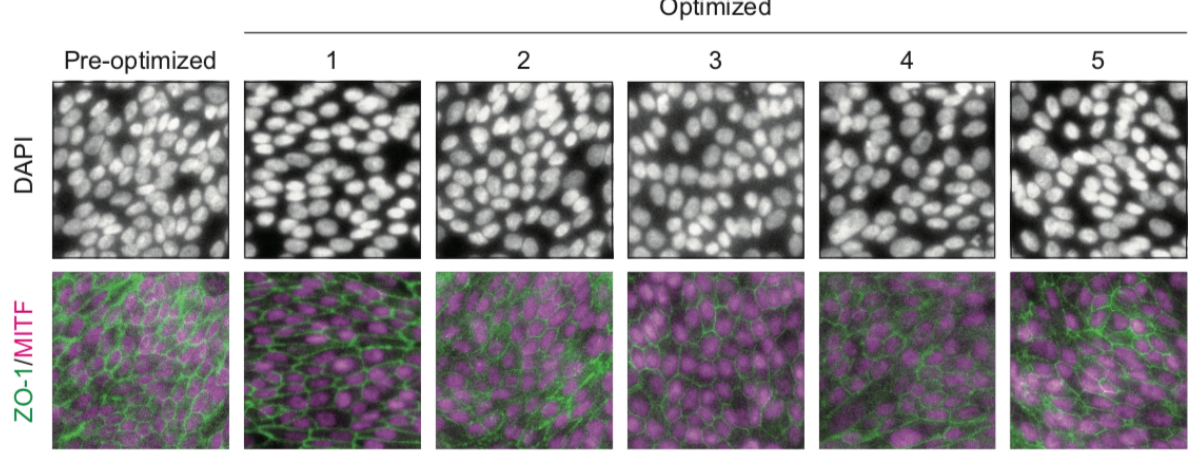

Figure 7. Quality evaluation of robot-induced RPE cells

A, Pigmentation score evaluation of the pre-optimized $(n=3)$ and top 5 conditions $(n=3$ each) from round 3 . Error bars represent the standard error of the mean (SEM). The numbers $1-5$ in the optimized group represent the first to fifth place conditions for round 3 (Figure 5C). Circles represent an individual score, bars represent the mean score, and error bars represent the SEM. Statistical significance was examined using two-way ANOVA and SNK post-hoc test. $P<0.05$ was considered significant. ${ }^{* * *} P<$ 0.001 versus pre-optimized. All other combinations detected no statistical significance.

B, Representative pigmented images of the pre-optimized and five optimized iPSC-RPE cells. Images acquired on DDay 34. ID labelling on the bottom reads 'V (validation) - Plate No. - Well No.'.

C-F, Cell biological analysis. After DDay 34, cells were purified, stocked, initiated, maintained for 4 weeks, and analyzed.

C, Examination of representative marker gene expression in RPE cells using RT-PCR. iPSC, undifferentiated iPSC; H-RPE (Lonza), Clonetics H-RPE (Lot \#493461, Lonza, USA); pre-optimized and optimized, LabDroid-induced RPE.

D-E, Quantification of representative secreted proteins from iPSC-RPE cells using ELISA. The supernatants were collected and the amount of VEGF (E) and PEDF (F) in the culture medium was analyzed $24 \mathrm{~h}$ after medium exchange ( $\mathrm{n}=3$ wells each). Circles represent an individual score, bars represent the mean score, and error bars represent the SEM. n.d. = not detected.

F, Co-staining of ZO-1 (green) and MITF (magenta) using immunohistochemistry. Nuclei were stained with DAPI. 